\title{
Recent developments in supernova research with VLBI
}

\author{
Norbert Bartel ${ }^{1}$ and Michael F. Bietenholz ${ }^{1,2}$ \\ ${ }^{1}$ York University, Toronto, M3J 1P3 Canada \\ email: bartel@yorku.ca \\ ${ }^{2}$ Hartebeesthoek Radio Observatory, PO Box 443, Krugersdrop 1740, South Africa \\ email: mbieten@yorku.ca
}

\begin{abstract}
Very long baseline interferometry (VLBI) observations during the last 30 years have resolved many supernovae and provided detailed measurements of the expansion velocity and deceleration. Such measurements are useful for estimating the radial density profiles of both the ejecta and the circumstellar medium left over from the progenitor. VLBI measurements are also the most direct way of confirming the relativistic expansion velocities thought to occur in supernovae associated with gamma-ray bursts. Well-resolved images of a few supernovae have been obtained, and the interaction of the ejecta as it expands into the circumstellar medium could be monitored in detail. We discuss recent results, for SN 1979C, SN 1986J, and SN 1993J, and note that updated movies of the latter two of the supernovae from soon after the explosion to the present are available from the first author's personal website.
\end{abstract}

Keywords. techniques: high angular resolution, radio continuum: stars, supernovae: general, supernovae: individual (SN 1979C, SN 1986J, SN 1993J), stars: mass loss, stars: neutron, galaxies: distances and redshifts.

\section{Introduction}

Radio emission of supernovae originates from the interaction of the ejecta with the circumstellar medium (CSM) left over from the massive progenitor star. It could also originate from the interior region close to the stellar corpse generated in the explosion. Very long baseline interferometry (VLBI) observations of a supernova can provide us with detailed information about the supernova's size, expansion velocity, deceleration, age, and morphology.

The characteristics of the expansion can be used to estimate density profiles of the ejecta and sometimes bounds on the mass-loss-to-wind-velocity ratio, $\dot{M} / w$, of the progenitor star, independent of radio-lightcurve fitting. When combined with velocities obtained from optical spectra, they can also be used in determining the geometrical distance to the supernova and its host galaxy. Further, they are the most direct way of confirming or disputing relativistic expansion velocities expected in supernovae related to gammaray bursts. The morphology gives us information on the clumpiness of the CSM and/or possibly the ejecta. The thickness of the radio-emitting shell region, thought to be between the forward and reverse shocks, is in turn related to the density profiles. Any emission from the central region of the image of the supernova could be related to particularly dense condensations in the shell itself, to an interior shell or to the stellar corpse expected to be left over after the explosion of the massive star. In Fig. 1 we show on the left side part of a radio image of a supernova and on the right side a sketch of a supernova model to facilitate interpretation of observations. 


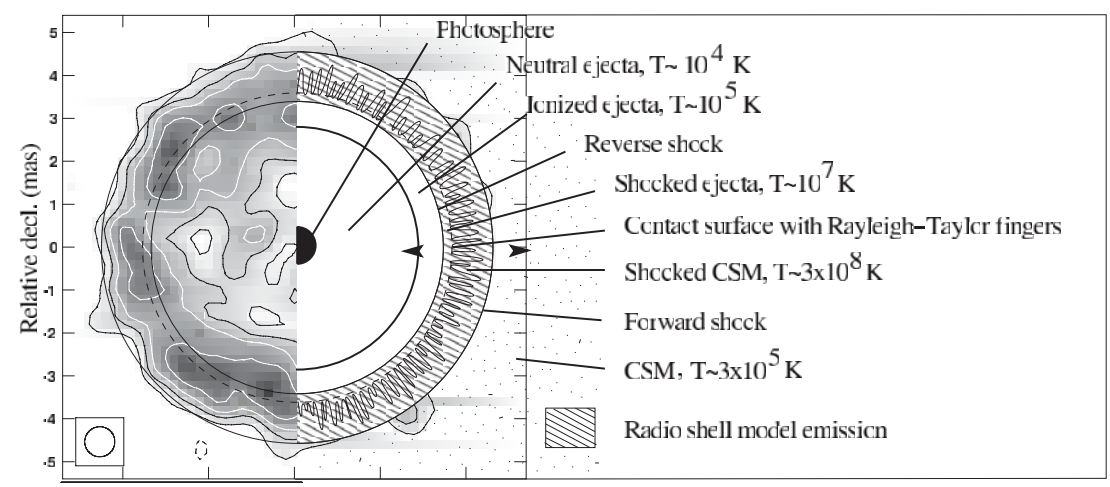

Figure 1. Left side: Composite VLBI image of SN 1993J at $8.4 \mathrm{GHz}$ taken at three epochs between 1998 and 2000. Circles indicate fit inner and outer radii of the shell. Right side: Sketch of the standard model of a core-collapse supernova interacting with the CSM and matched to the radio image. It shows different regions with typical temperatures. Radio emission is expected to emanate between the forward and the reverse shock (taken from Bartel et al. 2007).

Since the first VLBI observations of a supernova (SN 1979C) in 1982 (Bartel et al. 1985), many supernovae have been detected with VLBI, but only five of them could be imaged in detail. These are SN 1979C in M100 (Bartel \& Bietenholz 2008), SN 1986J in NGC 891 (Bietenholz et al. 2010a), SN 1987A in the LMC (Ng et al. 2011) and SN 1993J in M81 (Bietenholz et al. 2003; Bietenholz et al. 2010b; Marcaide et al. 2009a), all optically identified, and SN 2008iz in the highly optically opaque central region of M82 (Brunthaler et al. 2010a). In the remainder we discuss recent results on three of these supernovae.

\section{Supernova VLBI}

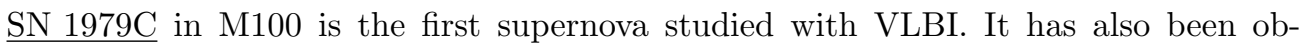
served for the longest time and showed almost free expansion for two decades (Bartel \& Bietenholz 2003; Marcaide et al. 2009b).

That was surprising. With a limit of the kinetic energy of $10^{51}$ erg for the shocked ejecta and the CSM shells it was suggested that $\dot{M}_{w} / w \sim 1 \times 10^{-5} M_{\odot} \mathrm{yr}^{-1}$ per $w=$ $10 \mathrm{~km} \mathrm{~s}^{-1}$, an order of magnitude smaller than estimated from radio-lightcurve fitting. However, strong deceleration is expected to start soon. In Fig. 2 (left) we show an image of SN 1979C. This image showed for the first time that the morphology was a shell. Despite the supernova's age, there was no indication of a central source above a limit of 15 times the $5 \mathrm{GHz}$ spectral luminosity of the Crab Nebula (Bartel \& Bietenholz 2008).

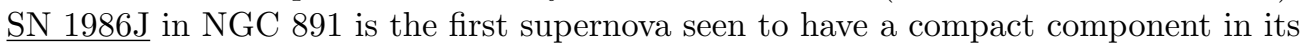
projected center (Bietenholz et al. 2004, Fig. 2 right;). The component was first seen in 2003 in images at 8 and $15 \mathrm{GHz}$, but was not seen at $5 \mathrm{GHz}$. Before that an inversion in the integrated spectrum above $8 \mathrm{GHz}$ had already been seen in 1999. Currently the compact component dominates images even at $5 \mathrm{GHz}$, and the spectral inversion has moved to lower frequencies. The component has a small proper motion of $1500 \pm 1500 \mathrm{~km} \mathrm{~s}^{-1}$, is marginally resolved at $22 \mathrm{GHz}$, and has an optically-thin spectral index indistinguishable from that of the shell. Three interpretations have been suggested. The component could be a pulsar wind nebula, with a spectral luminosity at $5 \mathrm{GHz} \sim 15$ times that of the Crab Nebula, or emission from close to the black hole left over from the explosion. It could also be part of the shell and be caused by the shock front imparting on a dense condensation 

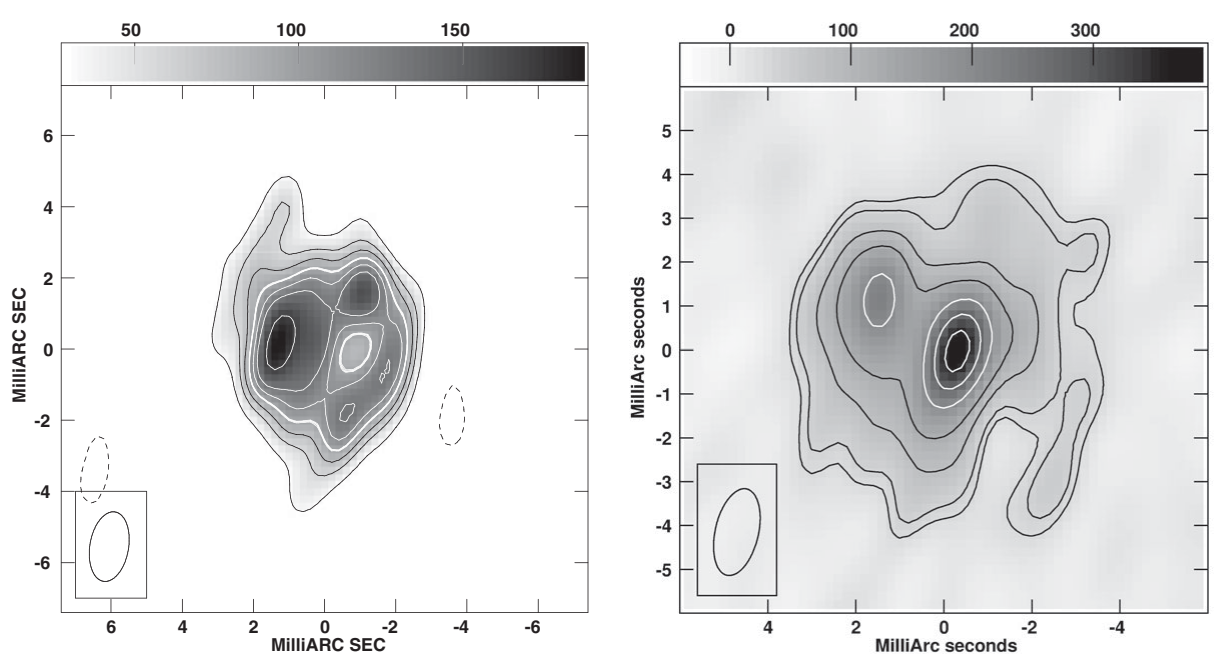

Figure 2. Left: VLBI image of SN $1979 \mathrm{C}$ at $5.0 \mathrm{GHz}$ at epoch 2005 February 25. The contour levels are at $-17,17,30,40,50$ (first white contour), 60,70 , and $90 \%$ of the peak brightness of $186 \mu \mathrm{Jy} \mathrm{beam}^{-1}$. The scale at the top is in $\mu \mathrm{Jy} \mathrm{beam}^{-1}$. Here and hereafter the FWHM of the beam is given in the left corner (taken from Bartel \& Bietenholz 2008). Right: VLBI image of SN 1986J at $5.0 \mathrm{GHz}$ at epoch 2008 October 25 . The contour levels are at $-7,7,10,20,30,40$, 50 (first white contour), 70 , and $90 \%$ of the peak brightness of $388 \mu \mathrm{Jy}^{\text {beam }}{ }^{-1}$. The scale at the top is in $\mu \mathrm{Jy}_{\text {beam }^{-1}}$ (Bietenholz et al. 2010a).

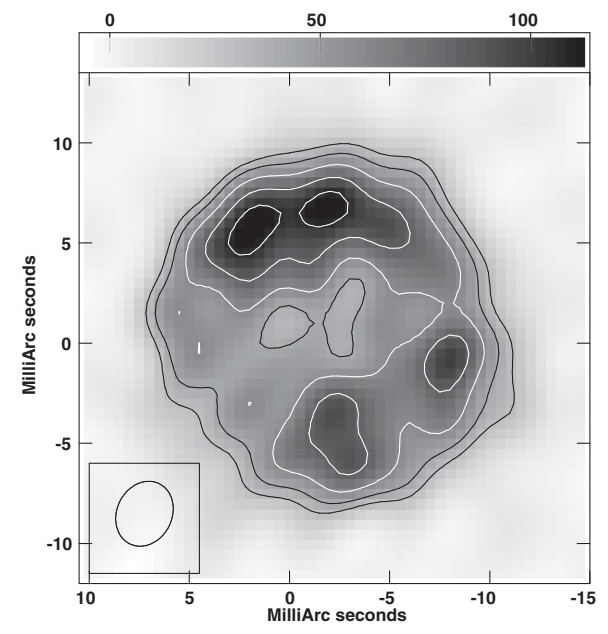

Figure 3. The latest VLBI image of SN 1993J, at $1.7 \mathrm{GHz}$, at epoch 2010 March 6 . The contours are at $25,35,50$ (first white contour), 70 , and $90 \%$ of the peak brightness of $117 \mu \mathrm{Jy} \mathrm{beam}^{-1}$. The scale at the top is in $\mu \mathrm{Jy}_{\text {beam }}{ }^{-1}$. The coordinate origin is within $64 \mu \mathrm{as}(\mathrm{rms})$ at the explosion center (taken from Bietenholz et al. 2010b).

of the CSM $\sim 1000$ times denser than the corresponding average CSM and fortuitously close to the projected center of the shell (Bietenholz et al. 2010a). Or the component is associated with the interaction of the shock with the slower parts of a highly anisotropic CSM formed in a binary system where the companion, left over from an earlier supernova explosion, has spriralled into the envelope of the progenitor of the observed supernova (Chevalier 2012). SN 2008iz also showed a compact central component but it has dimmed and is no longer visible in the latest images (Brunthaler et al. 2010b). 


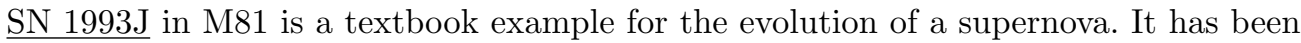
imaged from a month after the explosion to the present. The explosion occurred at a location we pin-pointed with $160 \mathrm{AU}$ accuracy in the galactic reference frame of M81. From there, the SN expanded isotropically to within 5.5\% (Bietenholz et al. 2001). Initially, the supernova expanded rapidly and almost freely with a velocity of $\sim 18,000 \mathrm{~km} \mathrm{~s}^{-1}$ and the radius $r \propto t^{m(t)}$, where $t$ is time and $m(t)=0.919 \pm 0.019$. Then the deceleration grew to $m(t)=0.781 \pm 0.009$ at $t \sim 1000 \mathrm{~d}$, and the velocity slowed down to $\sim 8500 \mathrm{~km} \mathrm{~s}^{-1}$.

The shell structure changed systematically, and the supernova swept up $\sim 0.3 M_{\odot}$, about equal to that of the small hydrogen shell thought to have remained around the progenitor after mass transfer to the binary companion. Subsequently the deceleration changed again, (Bartel et al. 2002, see also Martí-Vidal et al. 2011a,b) similarly to what was predicted from hydrodynamic simulations (Mioduszewski et al. 2001).

The transverse deceleration measured in the radio is very similar to the radial one measured in the optical. Using the expanding shock front method (ESM), we determined the distance to SN 1993J and M81: $3.96 \pm 0.06$ (stat.) \pm 0.29 (sys.) Mpc, suggesting $H_{0}=$ $66 \pm 11 \mathrm{~km} \mathrm{~s}^{-1} \mathrm{Mpc}^{-1}$ (Bartel et al. 2007).

Our latest image, from 2010, is displayed in Fig. 3. The supernova shell remains quite circular. The brightness is modulated around the rim, and the pattern of modulation has remained fairly similar over the last few years, and is therefore likely intrinsic rather than due to noise or deconvolution errors. A recent development is that the shell thickness is increasing rapidly (Bietenholz et al. 2010b), whereas only a slow increase is predicted by hydrodynamic simulations. This thickening of the shell can be seen when our latest images are compared with those from 1998 to 2000 (see Fig. 1) and earlier. In the last few years, the reverse shock has apparently moved rapidly away from the forward shock, and into the inner ejecta. No compact component has yet been found, with an upper limit on its spectral luminosity at $5 \mathrm{GHz}$ of 0.3 times that of the Crab Nebula.

\section{References}

Bartel, N. \& Bietenholz, M. F. 2003, ApJ, 591, 301

-. 2008, ApJ, 682, 1065

Bartel, N., Bietenholz, M. F., Rupen, M. P., \& Dwarkadas, V. V. 2007, ApJ, 668, 924

Bartel, N., Rogers, A. E. E., Shapiro, I. I., et al. 1985, Nature, 318, 25

Bartel, N., Bietenholz, M. F., Rupen, M. P., et al. 2002, ApJ, 581, 404

Bietenholz, M. F., Bartel, N., \& Rupen, M. P. 2001, ApJ, 557, 770

-. 2003, ApJ, 597, 374

-. 2004, Science, 304, 1947

-. 2010a, ApJ, 712, 1057

Bietenholz, M., Bartel, N., Rupen, M. P., et al. 2010b, in 10th European VLBI Network Symposium and EVN Users Meeting: VLBI and the New Generation of Radio Arrays (Proceedings of Science), 57B

Brunthaler, A., Martí-Vidal, I., Menten, K. M., et al. 2010a, A\&̊A, 516, A27

Brunthaler, A., Martí-Vidal, I., Menten, K. M., et al. 2010b, in 10th European VLBI Network Symposium and EVN Users Meeting: VLBI and the New Generation of Radio Arrays (Proceedings of Science), 55B

Chevalier, R. A. 2012, ApJ (Letters), 752, L2

Marcaide, J. M., Martí-Vidal, I., Alberdi, A., et al. 2009a, A\&̊A, 505, 927

Marcaide, J. M., Martí-Vidal, I., Perez-Torres, M. A., et al. 2009b, A\&A, 503, 869

Martí-Vidal, I., Marcaide, J. M., Alberdi, A., et al. 2011a, A $\& A$, 526, A142

-. 2011b, AESA, 526, A143

Mioduszewski, A. J., Dwarkadas, V. V., \& Ball, L. 2001, ApJ, 562, 869

Ng, C.-Y., Potter, T. M., Staveley-Smith, L., et al. 2011, ApJ (Letters), 728, L15 


\section{Discussion}

CAPpellaro: I remember that $79 \mathrm{C}$ was also seen to flaten in optical, few years after explosion. They deduce a mass loss $10^{-4} M_{\odot} y r^{-1}$. Is this consistent?

BARTEL: Not quite, since our estimate is an order of magnitude lower.

RAY: Is it possible to break the degeneracy of $n$ \& s (ejecta density profile index \& CSM density profile index) from the VLBI data?

BARTEL: It is not possible analytically by assuming the standard model with self-similar expansion. But the changing thickness of the shell and the changing deceleration if that occurs, together with hydrodynamic simulations may give clues as to the density profiles of the ejecta and the CSM. 